\title{
Reliability of the STIR Sequence for Acute Type II Odontoid Fractures
}

\author{
F.D. Lensing, E.F. Bisson, R.H. Wiggins III, and L.M. Shah
}

\begin{abstract}
BACKGROUND AND PURPOSE: The STIR sequence is routinely used to assess acute traumatic osseous injury. Because the composition of the odontoid in older individuals may be altered with osteopenia and decreased vascularity, the STIR sequence may not accurately depict the acuity of an odontoid fracture. The purpose of this study was to evaluate the reliability of the STIR sequence to differentiate acute-versus-chronic type II odontoid fractures in older patients, particularly those with osteopenia.
\end{abstract}

MATERIALS AND METHODS: A retrospective review was performed for patients with type II odontoid fractures during a 10-year period with both CT and MR imaging performed within 24 hours of injury. Patients were paired with controls of similar ages and were grouped by age. The STIR images were evaluated in a blinded fashion for the presence of hyperintensity in the odontoid. Demographic and clinical characteristics were also recorded.

RESULTS: Seventy-five patients with type Il odontoid fracture and 75 healthy controls (mean and median age of 57 years) were identified. The sensitivity of STIR to detect fracture in the age group 57 years and older was significantly worse than that in the age group younger than 57 years ( $54 \%$ and $82 \%$, respectively; $P=.018)$.

CONCLUSIONS: Older patients, particularly those with osteopenia, may have acute odontoid injuries without corresponding STIR hyperintensity. Additionally, interobserver agreement in STIR interpretation decreases with increasing patient age. As such, in this patient population, in which the presence of bone marrow edema as an indicator of fracture acuity may impact therapeutic decisions, correlation with CT findings and clinical history is crucial.

$\mathbf{S}$ pine fractures in older patients following ground-level falls are common, with type II odontoid fractures occurring most frequently. ${ }^{1-3}$ Prompt and accurate diagnosis of these fractures is critical in patient management because they are associated with a high rate of nonunion in some patient populations, particularly if acuity is unrecognized. ${ }^{4}$

Treatment of type II odontoid fractures remains controversial. Some series have reported successful fracture healing with external mobilization alone in $37 \%-75 \%$ of patients. ${ }^{5,6}$ However, specific factors that have been shown to contribute to nonunion with orthosis alone include advanced patient age, increased degree and angulation of odontoid displacement $\left(4-6 \mathrm{~mm}\right.$ and $>10^{\circ}$ angu-

Received December 21, 2013; accepted after revision January 19, 2014.

From the Departments of Radiology (F.D.L., R.H.W., L.M.S.) and Neurosurgery

(E.F.B.), University of Utah Health Sciences Center, Salt Lake City, Utah.

Forrester D. Lensing and Erica F. Bisson are co-first authors.

Please address correspondence to Lubdha M. Shah, MD, Department of Radiology, University of Utah Health Sciences Center, 30 North, 1900 East, 1A071, Salt Lake

City, UT 84132-2140; e-mail: lubdha.shah@hsc.utah.edu

http://dx.doi.org/10.3174/ajnr.A3962 lation), and delayed treatment. ${ }^{4,7-9}$ While operative intervention may result in high rates of fracture healing, surgery may involve excess morbidity and mortality in elderly patients. ${ }^{10,11}$ Because fracture acuity is a critical factor in healing, both for nonoperative and surgical interventions, it is important to identify the odontoid fracture when advising patients on treatment recommendations. ${ }^{12}$

Currently, cervical spine trauma is most commonly evaluated with multidetector CT with sagittal and coronal reconstructions, which have improved delineation of fractures compared with plain radiographs. ${ }^{13,14}$ There is an increasing role for MR imaging, with its superior soft-tissue resolution, in the acute setting in patients with neurologic injury not only to evaluate the spinal cord but also to define areas of ligamentous injury that may relate to clinical instability. Although the routine evaluation of MR imaging of the cervical spine involves careful review of all sequences, the STIR sequence is relied on to reveal marrow edema related to an acute fracture. In addition to the increased detection of softtissue injury, the STIR sequence is exquisitely sensitive to bone marrow pathology. ${ }^{15-17}$ This feature may be useful in identifying the acuity of a fracture. With acute fractures in young healthy patients, there is increased bone marrow edema resulting in in- 

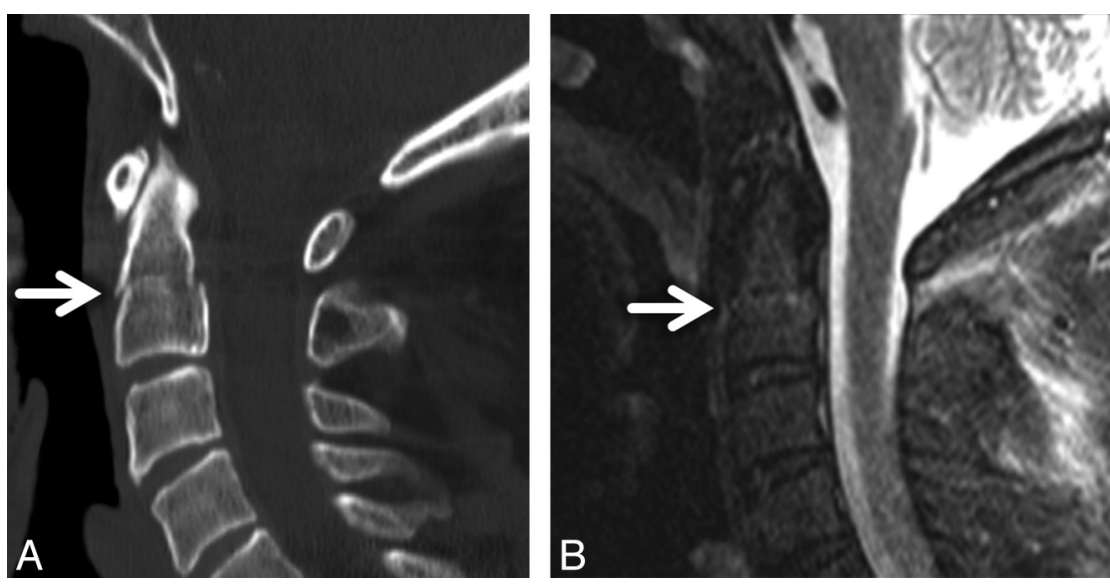

FIG 1. Sagittal CT reconstruction $(A)$ and sagittal STIR $(B)$ images from a 65-year-old man after a ground-level fall demonstrate a minimally displaced fracture at the odontoid base (white arrow) with minimal hyperintensity on the STIR sequence (white arrow).
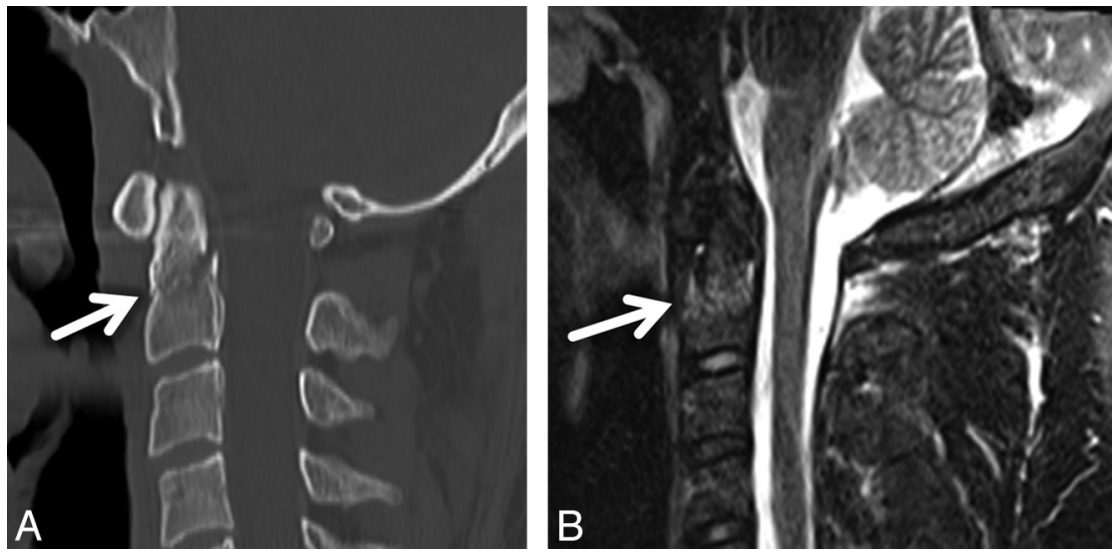

FIG 2. Sagittal CT reconstruction $(A)$ and sagittal STIR (B) images from a 37-year-old man after a motor vehicle collision show a minimally displaced fracture at the odontoid base (white arrow) with robust STIR hyperintensity in the bone marrow (white arrow).

traosseous STIR hyperintensity, ${ }^{15,18}$ which is often used in clinical decision-making as a marker of acuity. In contradistinction, in older patients with osteopenia, vertebral body fractures have been characterized by focal areas of enchondral bone formation adjacent to avascular necrotic bone and unreactive marrow. ${ }^{19}$ Because older patients, particularly those with osteopenia, have a more heterogeneous composition of the odontoid process with known decreased vascularity, we hypothesized that STIR hyperintensity would be less reliable as an indicator of the acuity of these fractures in this population.

\section{MATERIALS AND METHODS}

After approval by the institutional review board, a retrospective query of the radiology information system at a level 1 trauma center for the terms "odontoid fracture," "dens fracture," and/or "C2 fracture" during a 10-year period from 2002 to 2012 was performed. We identified patients presenting to the emergency department with a history of acute trauma and documented acute type II odontoid fracture on the basis of clinical history and CT evaluation who subsequently had MR imaging of the cervical spine. The same number of control patients with similar ages, also presenting with a history of acute trauma but without docu- mented C2 fracture by both CT and MR imaging, was also identified. Although these patients may have presented with trauma at other locations, this study focused solely on the $\mathrm{C} 2$ trauma. CT of the cervical spine was performed per trauma protocol when the patient was initially brought to the hospital. MR imaging of the cervical spine was performed within 12 hours of CT. All patients with C2 fractures were included in the analysis. Because patients' complex histories are often not known at the time of trauma, patients and controls were not excluded on the basis of comorbidities. In the retrospective review of these cases, all $\mathrm{C} 2$ fractures were considered secondary to trauma, not secondary to other unrelated pathologies.

\section{Imaging and Evaluation}

All MR imaging was performed on a 1.5T scanner (either Avanto or Aera; Siemens, Erlangen, Germany). The STIR sequence was performed by using $\mathrm{TI}=180, \mathrm{TE}=55 \mathrm{~ms}, \mathrm{TR}=4000 \mathrm{~ms}$, matrix $=256 \times 192 \mathrm{~mm}, \mathrm{FOV}=220 \times$ $220 \mathrm{~mm}$, and voxel size $=0.9 \times 0.9 \times 3$ $\mathrm{mm}$. Two senior neuroradiologists with Certificates of Added Qualification, each with $>10$ years of experience, independently evaluated the midline sagittal STIR sequences of all patients for the presence or absence of STIR hyperintensity, defined as nonlinear signal elevation within the odontoid (compared with the C3 vertebral body) (Figs 1 and 2).The images were presented to each reader in random order with the readers blinded to patient age and clinical history. If there was disagreement, a third reader, a spine-specialized neurosurgeon, made an independent assessment to break the tie; then, those images were reviewed with the neuroradiologists for a true consensus determination of STIR hyperintensity. Spine surgeons routinely use CT and MR imaging findings to make treatment decisions and often discuss their interpretation with the neuroradiologist, as was discussed in this study.

All CT was performed on a 64-section scanner (Somatom Definition AS Siemens, Erlangen, Germany) with 0.6-mm collimation and a helical acquisition. Sagittal and coronal 2.0-mm-thick images with 2.0-mm skip intervals were reconstructed from the axial data. All CT scans were evaluated for the presence or absence of acute fracture in the odontoid, by using features such as cortical disruption and irregular linear lucency without corticated margins. The readers were blinded to the CT results during their interpretation of the STIR images.

\section{Statistical Analysis}

The accuracy of using STIR hyperintensity to diagnose a fracture was measured by using a diagnostic test statistical approach. The 
Table 1: Presence of odontoid STIR hyperintensity in patients with acute fractures younger than $\mathbf{5 7}$ years of age

\begin{tabular}{lccc}
\hline Acute Fracture & STIR Present & STIR Absent & Total \\
\hline Present & 22 & 5 & 27 \\
Absent & 4 & 39 & 43 \\
Total & 26 & 44 & 70 \\
\hline
\end{tabular}

reference standard was fracture (either present or absent) as determined by CT and clinical history. The diagnostic test was STIR hyperintensity (either present or absent). The sensitivity and specificity were reported, along with $95 \%$ confidence intervals. To determine whether the diagnostic accuracy varied by age, we first dichotomized the patient age at the total sample median age of 57 years (into 2 groups: younger than 57 years and 57 years and older). Then a $2 \times 2$ cross-tabulation of fracture and STIR hyperintensity was formulated for each age subgroup. Next, in order to compare the sensitivity measured separately for the 2 age subgroups, the sensitivity row (fracture present cases) was taken from each table and combined them into a new $2 \times 2$ table, which was then tested for statistical significance by using a $\chi^{2}$ test, or Fisher exact test if any expected cell frequency was $<5 .{ }^{20} \mathrm{~A}$ similar approach was used for the specificity comparison, by combining the specificity row of each age subgroup into a $2 \times 2$ table. All reported $P$ values are for a 2 -sided comparison. Interrater reliability was measured with a $\kappa$ coefficient and was reported with an accompanying 95\% confidence interval. Statistical analysis was performed by using STATA (Version 12; StataCorp, College Station, Texas).

\section{RESULTS}

There were 75 patients ( 40 men and 35 women, median age of 67 years) with acute type II odontoid fractures found by clinical history and CT evaluation. These patients were then combined with 75 control patients of similar ages ( 41 men and 34 women, median age of 55 years) presenting with a history of acute trauma without documented C2 fracture. Dividing the subjects into 2 groups based on the overall mean and median age of 57 years, with 70 subjects in group 1 (younger than 57 years of age) and 80 in group 2 (57 years of age or older), with mean \pm SD age of $37.29 \pm$ 12.98 years and 73.05 ( $\mathrm{SD} \pm 10.55)$ in the groups, respectively.

For both readers, odontoid bone marrow STIR hyperintensity was more frequently observed in patients with acute fractures than in the nonfractured control subjects across all ages. The sensitivity of the STIR sequence for acute fracture was $88.9 \%$ (95\% CI, 77.4-95.8) and the specificity was $71.9 \%$ (95\% CI, 61.880.6). The sensitivity of STIR to detect fracture in the age group 57 years and older was significantly worse than that in the age group younger than 57 years (sensitivity $54 \%$ and $82 \%$, respectively; $P=$ .018). The specificity of negative STIR in the age group 57 years and older without fracture was similar to that in the age group younger than 57 years without fracture (specificity $94 \%$ and $91 \%$, respectively; $P=0.63$ ) (Tables 1 and 2 ).

There was substantial interobserver agreement between readers in the detection of STIR signal in patients younger than 57 years of age with type II odontoid fracture $(\kappa=0.79$; 95\% CI, $0.64-0.94)$. In subjects 57 years of age and older with odontoid fracture, there was moderate interobserver agreement in the evaluation of STIR signal intensity $(\kappa=0.50 ; 95 \% \mathrm{CI}, 0.30-0.69) .{ }^{21}$
Table 2: Presence of odontoid STIR hyperintensity in patients with acute fractures $\mathbf{5 7}$ years of age and older

\begin{tabular}{lccc}
\hline Acute Fracture & STIR Present & STIR Absent & Total \\
\hline Present & 26 & 22 & 48 \\
Absent & 2 & 30 & 32 \\
Total & 28 & 52 & 80 \\
\hline
\end{tabular}

\section{DISCUSSION}

Elderly patients are vulnerable to cervical spine injuries with the upper cervical spine $(\mathrm{C} 0-\mathrm{C} 2)$ being involved in $>50 \%$ of cases. $^{22-25}$ These injuries have an associated increased morbidity and mortality related to fracture nonunion and high comorbidity rates in this particular patient population. 2,26,27 Therefore, it is important to identify parameters that may play a role in fracture healing, including treatment recommendations, which are often based on the acuity of the fracture.

As MR imaging becomes more readily available and acquisition times become more rapid with newer scanners, MR imaging is being more frequently used in the acute trauma setting. The STIR sequence is very sensitive for the detection of osseous and soft-tissue edema as a result of fractures or microfractures ${ }^{28}$ and is routinely used to identify such edema in the cervical spine and other skeletal sites. ${ }^{15}$ One study showed STIR to be superior to CT in detecting subtle fractures, such as insufficiency fractures. ${ }^{29}$

Our data suggest that the STIR sequence, while sensitive for the detection of acute type II odontoid fracture in patients younger than 57 years, is significantly less sensitive in older patients, particularly those with osteopenia. Additionally, as patient ages increase, the agreement between observers evaluating STIR hyperintensity in acute type II odontoid fractures decreases from substantial to only moderate agreement.

We speculate that the decrease in sensitivity of STIR to detect osseous edema in the presence of acute type II odontoid fracture in older and osteopenic patients may relate to the unique anatomic and histologic changes that occur in the odontoid with senescence and with osteopenia. Angiographic studies in cadavers have suggested a watershed zone at the odontoid base between the anterior ascending and posterior ascending arteries. ${ }^{30}$ This area of decreased vascularity may account, in part, for the increased frequency of type II odontoid fractures in this population. ${ }^{31,32}$ In addition, the odontoid is located intrasynovially and, as such, does not receive periosteal blood supply. ${ }^{33}$ The combination of the vascular watershed and the lack of a periosteal blood supply contributes to overall poor vascularity in the odontoid, which contributes to the absence of bone marrow edema and, subsequently, the absence of STIR hyperintensity in the setting of acute fracture in the older population.

Osteopenia, characterized by decreased bone mineral attenuation with microarchitectural deterioration, is also a contributory factor in the occurrence of odontoid fractures. ${ }^{4}$ In addition to its tenuous blood supply, the odontoid base has a zone of decreased trabecular volume, decreased trabecular interconnection, and decreased cortical thickness. ${ }^{34,35}$ The zone of decreased trabeculation at the odontoid base undergoes substantial changes in the setting of osteoporosis, losing $64 \%$ of its bone mass compared with the adjacent $\mathrm{C} 2$ vertebral body and the tip of the odontoid. ${ }^{35}$ Therefore, osteopenia decreases the likelihood of bone marrow 
edema and consequent paucity of STIR hyperintensity in the setting of acute type II odontoid fracture in this patient population.

Although bone mineral densitometry was not available to categorize our subjects as osteoporotic, the literature has shown a significant correlation between a decreased bone mineral attenuation and advanced patient age. ${ }^{36,37}$ Gradual loss of skeletal mass begins in women in the fourth decade and accelerates following menopause. In men, bone loss begins in the fifth and sixth decades. There is no exact age that osteoporosis begins because there are multiple cofactors that can bring on an early onset of osteoporosis. Fifty-four percent of postmenopausal white women are estimated to have osteopenia, and 30\% have osteoporosis in at least 1 skeletal site. ${ }^{38}$ The general prevalence of osteoporosis rises from $5 \%$ among women 50 years of age to $50 \%$ at 85 years of age; among men, the comparable figures are $2.4 \%$ and $20 \%$, respectively. ${ }^{39}$

Despite the relative frequency of odontoid fractures in the elderly, there has been a lack of consensus regarding the optimal management of these fractures. Recent data, however, show level 2 evidence for the recommendation of surgical stabilization of acute type II odontoid fractures in patients 50 years of age or older, ${ }^{40}$ with higher union rates described with operative management in elderly patients. ${ }^{37,41-43}$ A recent multicenter study showed high rates of mortality and nonunion in nonoperatively treated type II odontoid fractures in the elderly. ${ }^{12}$ As such, there is an increasing tendency toward the surgical treatment in the literature. ${ }^{11,44}$ Because MR imaging and the STIR sequence, in particular, are used to determine fracture acuity and, therefore, treatment recommendations, it is important that we understand the limitations of this sequence. On the basis of our results, we speculate that STIR signal hyperintensity in the odontoid process may reflect a surrogate marker of vascularity and the presence of normal bone marrow composition.

This study has some limitations. First, the retrospective design may have introduced bias in suspecting fracture, despite the randomization with controls of similar ages. This may have contributed to the variability in the detection of STIR signal between readers. The focus of this article is the detection of STIR hyperintensity in odontoid fractures, particularly type II given the common occurrence of this type of injury. We did not assess the presence of additional fractures, which can occur with odontoid fractures. Although the readers were limited to the sagittal midline STIR image for the evaluation of signal intensity, it is conceivable that edema in the $\mathrm{C} 1$ body and/or the adjacent soft tissues may have influenced the interpretation.

Second, there were false-positive results (ie, STIR hyperintensity without fracture) in both age groups, but they were higher in the younger group. These are likely due to the subtly increased STIR signal intensity around the hypointense dentocentral synchondrosis (simulating a fracture cleft) at the odontoid base, due to a persistent cartilaginous matrix (Fig 3). ${ }^{45}$

Third, our MR imaging scans were obtained on a 1.5T Siemens system, and because imaging parameters may vary depending on the field strength, our results may not be generalizable across different manufacturers and different imaging protocols. Increased magnetic susceptibility from bony trabeculae has been described

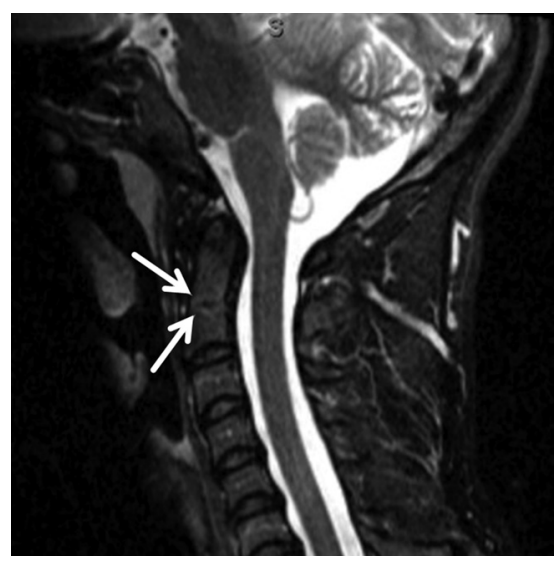

FIG 3. Sagittal STIR image of a 32-year-old female trauma patient shows slight STIR hyperintensity around the hypointense dentocentral synchondrosis (simulating a fracture cleft) at the odontoid base, due to persistent cartilaginous matrix (white arrows).

at 3T, possibly resulting in lower signal intensity on T2-weighted and STIR imaging. ${ }^{15}$

Last, we did not exclude studies on the basis of motion artifacts. Motion artifacts may limit the diagnostic utility of MR imaging, and the STIR sequence has been shown to be particularly sensitive to patient motion. ${ }^{17,46}$ While our readers did not comment on the quality of the images, it is possible that relatively minor patient motion could have masked subtle STIR signal elevation in the bone marrow.

\section{CONCLUSIONS}

The STIR signal elevation is relatively insensitive for the presence of an acute type II odontoid fracture in patients 57 years of age and older, particularly in those patients with osteopenia. Furthermore, interobserver agreement for the detection of the STIR signal abnormality declined in older patients compared with younger patients. These findings suggest that in older patients, possibly with osteopenia, with an acute odontoid fracture, the STIR sequence is limited in its detection of bone marrow edema and, therefore, should be interpreted with caution.

Disclosures: Erica F. Bisson-UNRELATED: Grants/Grants Pending: Sorenson Foundation, ${ }^{*}$ Margolis Foundation. * *Money paid to the institution.

\section{REFERENCES}

1. Lomoschitz FM, Blackmore CC, Mirza SK, et al. Cervical spine injuries in patients 65 years old and older: epidemiologic analysis regarding the effects of age and injury mechanism on distribution, type, and stability of injuries. AJR American $J$ Roentgenol 2002;178:573-77

2. Malik SA, Murphy M, Connolly P, et al. Evaluation of morbidity, mortality and outcome following cervical spine injuries in elderly patients. Eur Spine J 2008;17:585-91

3. Chutkan NB, King AG, Harris MB. Odontoid fractures: evaluation and management. J Am Acad Orthop Surg 1997;5:199-204

4. Ryan MD, Taylor TK. Odontoid fractures in the elderly. J Spinal Disord 1993;6:397-401

5. Polin RS, Szabo T, Bogaev CA, et al. Nonoperative management of Types II and III odontoid fractures: the Philadelphia collar versus the halo vest. Neurosurgery 1996;38:450-56, discussion 456-57

6. Wang GJ, Mabie KN, Whitehill R, et al. The nonsurgical management of odontoid fractures in adults. Spine (Phila $\mathrm{Pa}$ 1976) 1984;9:229-30 
7. Greene KA, Dickman CA, Marciano FF, et al. Transverse atlantal ligament disruption associated with odontoid fractures. Spine 1994; 19:2307-14

8. Lennarson PJ, Mostafavi H, Traynelis VC, et al. Management of type II dens fractures: a case-control study. Spine (Phila $\mathrm{Pa}$ 1976) 2000;25:1234-37

9. Lind B, Nordwall A, Sihlbom H. Odontoid fractures treated with halo-vest. Spine 1987;12:173-77

10. Geisler FH, Cheng C, Poka A, et al. Anterior screw fixation of posteriorly displaced type II odontoid fractures. Neurosurgery 1989;25: 30-37, discussion 37-38

11. Campanelli M, Kattner KA, Stroink A, et al. Posterior C1-C2 transarticular screw fixation in the treatment of displaced type II odontoid fractures in the geriatric population: review of seven cases. Surg Neurol 1999;51:596-600, discussion 600-01

12. Smith JS, Kepler CK, Kopjar B, et al. The effect of type II odontoid fracture nonunion on outcome among elderly patients treated without surgery: based on the AOSpine North America Geriatric Odontoid Fracture study. Spine (Phila Pa 1976) 2013;38:2240-46

13. Sheikh K, Belfi LM, Sharma R, et al. Evaluation of acute cervical spine imaging based on ACR Appropriateness Criteria(R). Emerg Radiol 2012;19:11-17

14. Gale SC, Gracias VH, Reilly PM, et al. The inefficiency of plain radiography to evaluate the cervical spine after blunt trauma. J Trauma 2005;59:1121-25

15. Shah LM, Hanrahan CJ. MRI of spinal bone marrow. Part I. Techniques and normal age-related appearances. AJR Am J Roentgenol 2011;197:1309-21

16. Alyas F, Saifuddin A, Connell D. MR imaging evaluation of the bone marrow and marrow infiltrative disorders of the lumbar spine. Magn Reson Imaging Clin N Am 2007;15:199-219, vi

17. Mirowitz SA, Apicella P, Reinus WR, et al. MR imaging of bone marrow lesions: relative conspicuousness on $\mathrm{T} 1$-weighted, fat-suppressed T2-weighted, and STIR images. AJR American J Roentgenol 1994;162:215-21

18. Long SS, Yablon CM, Eisenberg RL. Bone marrow signal alteration in the spine and sacrum. AJR American J Roentgenol 2010;195: W178-200

19. Antonacci MD, Mody DR, Rutz K, et al. A histologic study of fractured human vertebral bodies. J Spinal Disord Tech 2002;15:118-26

20. Pepe MS. The Statistical Evaluation of Medical Tests for Classification and Prediction. Oxford, UK: Oxford University Press; 2003

21. Landis JR, Koch GG. The measurement of observer agreement for categorical data. Biometrics 1977;33:159-74

22. Lieberman IH, Webb JK. Cervical spine injuries in the elderly. J Bone Joint Surg Br 1994;76:877-81

23. Müller EJ, Wick M, Russe O, et al. Management of odontoid fractures in the elderly. Eur Spine J 1999;8:360-65

24. Weller SJ, Malek AM, Rossitch E Jr. Cervical spine fractures in the elderly. Surg Neurol 1997;47:274-80, discussion 280-81

25. Ryan MD, Henderson JJ. The epidemiology of fractures and fracture-dislocations of the cervical spine. Injury 1992;23:38-40

26. Irwin $\mathrm{ZN}$, Arthur M, Mullins RJ, et al. Variations in injury patterns, treatment, and outcome for spinal fracture and paralysis in adult versus geriatric patients. Spine 2004;29:796-802
27. Jackson AP, Haak MH, Khan N, et al. Cervical spine injuries in the elderly: acute postoperative mortality. Spine 2005;30:1524-27

28. Spiegl UJ, Beisse R, Hauck S, et al. Value of MRI imaging prior to a kyphoplasty for osteoporotic insufficiency fractures. Eur Spine J 2009;18:1287-92

29. Cabarrus MC, Ambekar A, Lu Y, et al. MRI and CT of insufficiency fractures of the pelvis and the proximal femur. AJR American J Roentgenol 2008;191:995-1001

30. Schiff DC, Parke WW. The arterial supply of the odontoid process. J Bone Joint Surg Am 1973;55:1450-56

31. Maiman DJ, Larson SJ. Management of odontoid fractures. Neurosurgery 1982;11:820

32. Elgafy H, Dvorak MF, Vaccaro AR, et al. Treatment of displaced type II odontoid fractures in elderly patients. Am J Orthop 2009;38: 410-16

33. Southwick WO. Management of fractures of the dens (odontoid process). J Bone Joint Surg Br 1980;62:482-86

34. Amling M, Hahn M, Wening VJ, et al. The microarchitecture of the axis as the predisposing factor for fracture of the base of the odontoid process: a histomorphometric analysis of twenty-two autopsy specimens. J Bone Joint Surg Br 1994;76:1840-46

35. Amling M, Posl M, Wening VJ, et al. Structural heterogeneity within the axis: the main cause in the etiology of dens fractures: a histomorphometric analysis of 37 normal and osteoporotic autopsy cases. J Neurosurg 1995;83:330-35

36. Lilley J, Eyre S, Walters B, et al. An investigation of spinal bone mineral density measured laterally: a normal range for UK women. Br J Radiol 1994;67:157-61

37. Reinhold M, Bellabarba C, Bransford R, et al. Radiographic analysis of type II odontoid fractures in a geriatric patient population: description and pathomechanism of the "Geier"-deformity. Eur Spine J 2011;20:1928-39

38. Woolf AD, Pfleger B. Burden of major musculoskeletal conditions. Bull World Health Organ 2003;81:646-56

39. Kanis JA, Johnell O, Oden A, et al. Risk of hip fracture according to the World Health Organization criteria for osteopenia and osteoporosis. Bone 2000;27:585-90

40. Ryken TC, Hadley MN, Aarabi B, et al. Management of isolated fractures of the axis in adults. Neurosurgery 2013;72(suppl 2):132-50

41. Frangen TM, Zilkens C, Muhr G, et al. Odontoid fractures in the elderly: dorsal C1/C2 fusion is superior to halo-vest immobilization. J Trauma 2007;63:83-89

42. Collins I, Min WK. Anterior screw fixation of type II odontoid fractures in the elderly. J Trauma 2008;65:1083-87

43. Dailey AT, Hart D, Finn MA, et al. Anterior fixation of odontoid fractures in an elderly population. J Neurosurg Spine 2010;12:1-8

44. Börm W, Kast E, Richter HP, et al. Anterior screw fixation in type II odontoid fractures: is there a difference in outcome between age groups? Neurosurgery 2003;52:1089-92, discussion 1092-94

45. Gebauer M, Lohse C, Barvencik F, et al. Subdental synchondrosis and anatomy of the axis in aging: a histomorphometric study on 30 autopsy cases. Eur Spine J 2006;15:292-98

46. Mirowitz SA. Fast scanning and fat-suppression MR imaging of musculoskeletal disorders. AJR American J Roentgenol 1993;161: 1147-57 